\title{
Ueber Kapselabhebungen.
}

\author{
Von \\ Dr. Alfred Topolanski, \\ Augenarzt im Barmherzigen Spital in Wien. \\ Hierzu Tafel XIII, Fig. 1-5.
}

Mit Kapselabhebung bezeichne ich jenen Zustand im Auge, bei welchem die Linsenkapsel mit der Linsensubstan\% nicht mehr ihren normalen, unmittelbaren Contact hat, sondern von dieser durch eine dazwischen liegende Schicht mit mehr oder weniger wässerigem Inhalt losgetrennt erscheint. Jene Fälle also, in welchen durch eine Veränderung des Inhaltes des Kapselsackes die Linse zu krümlichen, in Fliissigkeit aufgeschwemmten Massen umgewandelt wurde (auf deren Grund oft der Linsenkern herabgesunken ist) halte ich davon ausgeschlossen. Es gilt aur eine Continuitätstrennung der Kapsel von der Iinse.

I.

Die angeborene Kapselabhebung. In diesen Fällen zeigt die Linse das bekannte Bild der Linsencolobome als einen Defect an ihrer Randcontinuität in Form von Einkerbungen (oder auch nur einer solchen), iiber welche die Kapsel in ihrem normalen Situs hinwegzielht und so eine Art halbmondförmiger Haube über der Linse bildet. Sie scheint mit einer Flüssigkeit gefüllt zu sein, da auf ihr auch mit dem lichtschwachen Spiegel keinerlei Streifung wahrzunehmen ist, die freilich auch nur sehr schwer zu beobachten wäre. Der Linsenrand erscheint als beschattete Contour am Grunde der Haube. Es han- 
delt sich hier also um ein blasenartiges Kapselsegment, das dem Linsencolobom aufsitzt.

Ich habe einen solchen Fall gesehen, bei dem es sich um diese Form der Abhehung der Kapsel, um einen nicht mit Linsenmasse, sondem offenbar Flüssigkeit erfüllten Kapselabschnitt handelte und lese zu meinem Vergnügen, dass auch Th. Christen in einer Publication (Knapp's Archiv XXIX, Bd. 3-4, pag. 237) allerjüngsten Datums gleichfalls von einer solchen Beobachtung berichtet. $\mathrm{Er}$ sagt daselbst: „Besondere Erwähnung verdient das Verhalten der Kapsel in zwei Fällen, wo der Kapselsack den Linsenrand iiberragt und seine normale Stellung beibehält, obwohl die ausfüllende Linsenmasse fehlt ...." In den beobachteten Fällen handelte es sich um Individuen mittleren Alters, bei denen keine Linsentrübung dadurch belingt war. Es kommt also der Zustand, dass Kapsel und Linse von einander getrennt, der Zwischenraum zwischen heiden durch Flüssigkeit ausgefüllt ist, angeboren vor. Sehr richtig bemerkt Christen, dass dieses Verhältniss nicht unwichtig für die Frage der Abstammung der Kapsel ist; dieser Umstand würde damn für die alte Ansicht Arnold's sprechen, dass sie entstehe "durch Differenzirung des die Linse umhïllenden und von dem mittleren Keimblatte abstammenden Gewebes". Entscheidend aher in der Frage der Genesis der Kapsel kann dieser Befund wohl nicht sein, denn ob nicht an dem durch das Colobom anders gelagerten Linsenrande eine glasartige Kapsel als Ueberzug von den Epithelzellen ausgehend doch noch da ist, kann ohne anatomische Präparate nicht entschieden werden.

\section{II.}

a) Kapselabhebungen kommen auch in normalen Augen, die keinerlei Spuren irgend welcher Veränderungen weder durch Krankheit noch durch das Alter oder sonstwie zeigen, ror, und zwar am Rande der Linse. Auf dieses Verhalten 
habe ich gleichzeitig mit Magens hingewiesen und diese Fälle näher beschrieben (s. Klinische Monatsblätter f. Augenheilkunde, März 1892). Ich will daher darauf nicht näher eingehen, sondern nur in Kürze angeben, dass der Inhalt derselben im Anfangsstadium die Tinsensubstanz selbst ist, und dass erst bei einer gewissen Vergrösserung bei der Bildung der eigentlichen Abhebung dann ein Raum zwischen der Linse und der Kapsel frei wird, der sich auf Präparaten als mit Resten einer offenbar flüssigen Masse, in der sich kleine Krümelchen befinden, erfüllt zeigt. Diese Kapselabhebungen sind in der Form von der Ursache ihrer Entstehung - dem Zng der Zonula auf die Kapsel - abhängig und sind demnach zeltförmige Erheloungen und haben ihren Platz am Aequator der Linse resp. dem Zonulaansatze an der Kapsel. - Ich bin nicht im Stande zu sagen, ob diese Abhebungen stets ohne Bedeutung für die Linse sind, wie man nach dem äussern Ansehen glauben sollte, und auch Magens drückt sich nach dieser Richtung hin reservirt ans. Bei der Bedeutung des Ortes aber möchte man sie fast bei manchen Katarakten fïr nicht blos zufällige Erscheinungen. halten, und so komme ich dadurch auf die

b) zeltförmigen Erhebungen des Linsenrandes zu sprechen, die an dieser Stelle mitunter bei kataraktösen Linsen zu finden sind. Ich habe einige Zeit hindurch sehr gerne der eigentlichen Extraction kataraktöser Linsen eine priparatorische Iridektomie vorausgeschickt und dann oft zu sehen Gelegenheit gehabt, dass diese Erhebungen der Kapsel am Linsenrande gar nicht so sehr selten bei kataraktösen Linsen vorkommen, und auch wiederholt gesehen, dass streifenförmige Trübungen der Linse sehr geme vom Liusenrande her ihren Ursprung nehmen als Fortsetzung einer solchen Kapselabhebung an ihrem Rande.

Magnus („Entwicklung des Altersstaares“, Breslau 1892) spricht den Verdacht aus, dass die grossen Schläuche 
Furchungen der Linsensubstanz an ihrer Oberfläche sein könnten, und es würde sich nun um die Frage handeln, entstehen die zellförmigen Abhebungen gern dort, wo die Schläuche sind, oder umgekehrt die Schläuche dort, wo schon Abhebungen sind. Vielleicht gehört beides demselben Process an; es erscheint mir am wahrscheinlichsten. Hat eine senile Katarakt Schrumpfung eingegangen, so sieht man die Abhebung fast ausnahmslos. Sie hat dann wieder dieselbe Ursache in dem Widerstande der Zonula. Die geschrumpfte Cataracta senilis bildet auch den Uebergang für die Gruppe

\section{III.}

bei welcher es sich darum handelt, dass Abhebungen (nicht die eben erwähnten zellförmigen des Linsenäquators) an Linsenkapseln dadurch entstehen, dass in pathologisch veränderten Augen a) die Linse einen Schrumpfungsprocess mitmacht. Wird aus irgend einer Ursache in einem schwer kranken Auge eine triibe Linse in ilhrem Volumen zuriickgehen, so zieht sich der Kapselsack nicht immer entsprechend stark zusammen. Die Kapsel ist wohl sehr elastisch und wird sicher beim Schrumpfen einer Linse ein wenig in sich zusammen gehen, jedoch nur in geringem Maasse.

Wird bei einer Discission die Kapsel geschlitzt, so rollen sich ja immer die Ränder um, sie ist kein steifer Sack. Wird aber die Kapsel durch eine Anheftung festgehalten, sei es durch eine Irisverwachsung oder durch Fasern der Zonula, so bleibt sie in der Form noch als Sack bestehen, während die Linsenmasse zurückweicht und es entsteht dadurch ein mit Flüssigkeit gefüllter Raum zwischen beiden, die Kapsel selbst hebt sich in Falten ab. Ein Fall mag hier als Beispiel erwähnt werden.

Fin junger Mann von 17 Jahren erlitt in der Weise eine Verletzung, dass eine auf ihn zugeworfene $1^{1 / 2} \mathrm{~cm}$ grosse Heftnadel aus Stahl die Cornea nach aussen unten von der Mitte 
perforirte. Die Ixis prolabirte, die gleichfalls verletzte Linse war an die Hinterwand der Hornhaut angepresst. Ich machte nach innen zu die Iris los und schnitt sie heraus. An der Verletzungsnarbe blieb rückwärts ein Stückchen Iris angeheilt, sonst war der Pupillarrand frei beweglich (s. Fig. 1, Taf. XIII). Die Linse trübte siclı vorn im Umfange einer Hälfte des Radius zu einer weissen diclıten, wie inkrustirten Masse - das läufige Bild traumatischer linsentribung, - von welcher Masse aus sich trïbe Ausläufer in Nlammenform gegen die durchsichtig gebliebene Peripherie hin erstreekten. Anfangs blieb der ganze uibrige Cortex der Linse normal, der Abstand zwisehen diesen und den sichtbaren Ciliarfortsätzen gleichfalls. Allmählich in Verlaufe von Mfonaten jedoch trat eine leichte oberflächliche Trühung auch dieses linsentheiles ein, und der Alustand des Aequators von den Fortsätzen war ein entschieden viel breiterer, was nach einer genauen Zeichnung: constatirt werden konnte. Mit dem lichtschwachen Spiegel konnte man central vom Linsenäquator, der sich entrundete und zackige Kapselabhebungen, wie sie suh II beschrieben, zeigte, einen diesem parallel verlaufenden doppelt contourirten Saum beobacltten, der gleichfalls nicht vollkommen rund, sondern ausgezackt erschien und sich dureh seine Parallaxe mit der Umgebung und auch durch einen lichten Schatten lieim schiefen Beleuchten nach der andern Seite hin als Kapselabhebung documentirte. Es ist also durch einen Sehrumpfungsprocess in der pathologiseh veränderten Iinse an jener Stelle, wo die vorderen Zonulastränge auf die Kapsel hin fächerförmig ausstrallen, eine Abhebung in der ganzen Breite längs diesel' Ausstrahlungszone entstanden (s. Fig. 1, Taf. XIII).

b) Es kann ferner in einem pathologisch veränderten Auge die Bildung einer circumscripten Kapselkatarakt die Veranlassung zu einer Kapselabhebung werden, und zwar dann, wenn die Kapselkatarakt einen regressiven Process mitmacht und an Volumen abnimmt, so dass dadurch die Kapsel, deren Elasticität allein nicht melr hinreicht, um diesem Schrumpfungsprocesse zu folgen, ein Uebergewicht als Continens gegenüber dem zurückgegangenen Contentum erhält. Die Kapsel wird dann in Form von Blasen von der linse abgehoben bleiben. Die Kapselkatarakt an und für sich führt dadurch, dass sie entsteht 
und wächst, schon zu einer Kapselabhebung in dem Stadium ihres Anwachsens; insofern aber, als das Gewebe der Katarakt ja nur von dem Epithel abstammt und den Inhalt der abgehobenen Kapsel bildet, möchte ich da nicht so sehr Gewicht darauf legen, als vielmehr auf das spätere Stadium der Schrumpfung, die in diesen neugebildeten Geweben eintritt. Diese Verhältnisse der Schrumpfung sind von Becker in der Anatomie der gesunden und kranken Linse so erschöpfend beschrieben worden, dass ich mich wohl darauf beschränken darf, auf diese hinzuweisen.

Der Vollständigkeit wegen bringe ich hierzu die Figur 4 (Taf. XIII), welche uns zeigt, wie auf einem regressiv veränderten Kapselkataraktgewebe die Kapsel in Form von grösseren und kleineren Blasen unregelmässig aufliegt, die im Durchschnitt als Schlingen resp. Falten anzusehen sind. Dass jene glasartige Membran, welche die Kapselkatarakt nach innen zu umgiebt, nicht durch Spaltung der alten Linsenkapsel (Beeker), sondern als eine Neubildung aus dem Kapselepithel aufzufassen ist, geht aus den Befunden Wagenmanns und Versuchen C. Schloessers u. a. hervor. Es handelt sich also hier nicht um eine Lamellenahlebung.

Wieso die Schrumpfung und Abhebung der Kapsel von grosser Wichtigkeit für die weitere Lagerung der ganzen Linse sein kann, ist von vorne herein klar; ist nämlich der Zusammenhang der Linse resp. Kapsel mit dem Aufhängeapparat so sehr gelockert, dass dadurch ein Missverhältniss der Spamnung entsteht, so liegt es wohl nahe, spontane I uxationen der Linse, so selten sie auch vorkommen könuen, darauf zu beziehen, um so mehr als man ja sehen kann, dass in solcken Fällen die Erkrankungen, welche Ursache der Kapselkataraktbildung geworden sind, an den Ansatzstellen der Zonula auch diese selbst lockern und die Luxation unterstiätzen müssen - Verhältnisse, auf die ich ein anderes Mal zurückkommen will. 
IV.

Kapselabhebungen von Narben ausgehend. Nun kommen wir zu jener Gruppe von Fällen, bei welchen eine Narbe der Kapsel (und dem etwa angewachsenen Trisepithel) durch die Schrumpfung, welche sie ja wie eine Narbe der äussem Haut mitmacht, einen Zug ausiilbt und durch diesen eine Abhebung veranlasst. Da es hier nicht eine Verkleinerung des Kapselinhaltes ist, welche diese von der Linse sich trennen lässt, sondern ein auf sie ausgeübter Zug, so ist auch die Form eine andere, nämlich die von Falten, und zwar mit der Richtung vom Orte des Znges aus, also in der Art, dass sich die Faltungen über die Oberfläche hin erstrecken, von der Narbe weg auslaufend. Die Falten sind so beschaffen, dass sie an der Narbe eine breitere Basis haben und von dieser sich verschmälern und endlich in feinsten Spitzen sich verlieren. Sie können einzeln stehen, können aber auch in ganzen Büscheln neben einander von der Narbe ausgehen, bis zu 12 und dariber.

Beim Ansehen eines solchen Auges von vorne oder bei seitlicher Beleuchtung sind sie gewöhnlich nicht sichtbar, hingegen sind sie im durchfallenden Lichte bei lichtschwachem Spiegel sofort zu bemerken insbesondere dadurch, dass sie einmal als dunkle spitzige Figuren, das andere Mal bei einer Drehung des Spiegels gerade umgekehrt hellglänzend, stark Licht.reflectirend, wie Glassplitter, sich präsentiren. Fine geringe Bewegung mit dem Spiegel genügt, um diesen Wechsel zu veranlassen, so dass diese Stellen mitunter durch ihr Flimmern auffallen. Es sind mir Fälle dieser Art bei verschiedener Ursache von Narbenbildung vorgekommen. Ich will einige davon als besonders interessant anführen.

Ein Patient wurde wegen Glaukom von mir zuerst nach oben iridektomirt. Trotzdem die Iris sehr breit und bis zum Ansatze entfernt worden war, trat nach einiger Zeit Recidiv auf. Da Eserin vorübergehend Erfolg hatte, wiederholte ich die Iri- 
dektomie nach aussen. An jener Stelle der Linse (Fig. 2, Taf. XIII), wo der äussere Colobomschenkel der ersten Iridektomie angelagert war, zeigte sich nach der zweiten, dass eine Verklebung der lris mit der Kapsel stattgefunden hatte, da Irispigment haften geblieben war. Nach Verlauf von ca. 6 Wochen, während welcher Zeit ich eine Trübung der Narbenstelle bemerkte, konnte ich mit dem lichtschwachen Spiegel eine von dieser Stelle ausgehende kurze Falte gegen die Mitte der Linse zu entdecken, welcher sich in rascher Aufeinanderfolge innerhalb von ca. 14 Tagen eine grosse Anzahl gleicher anreihten, so dass schliesslich 9 kïrzere und längere faltige Abhebungen zu constatiren waren, bei welcher Anzahl es auch blieb. Sie boten jene eingangs erwähnten Merkmale und bestehen nun seit vielen Monaten, ohne dass die Linse in ihrem Aussehen hinter ihnen irgendwie eine Störung erfahren hätte; die übrige Linse ist nämlich durchsichtig. In wie weit sie sellsst auf das Sehvermögen Einfluss nehmen, kann ich nicht sagen, da eben das Auge auch sonst durch den Glaukomprocess schwer erkrankt war.

Nicht uninteressant war es, zu sehen, wie diese Narbe die Linse in ihrer Form beeinflusste. Die Narbe verlief nämlich (s. Fig. 2, Tat. XIII) zuerst entsprechend dem früheren Colobomschenkel gerade nach oben, dann nach aussen über die Linse, wo sie vor dem Linsenrande aufhörte. Der Linsenrand zeigte sich ron nomaler Rundung. Almählich aber und zwar nachdem sclon die Kapselabhebungen in ilrer jetzigen Form vorhanden waren, schien es, als wenn die Narbe gegen den Linsenrand hin sich fortgesetzt hätte, indem nämlich dort die Durchsichtigkeit flurch ein feines Bändchen, das offenbar ganz oberfiachlich war, unterbrochen wurde, und gleichzeitig begann der Linsenrand eine Einschnturung za zeigen. Ich halte auch das Bild, wie der Linsenrand es auf Fig. 2 (Taf. XIII) darstellt, durch eine Einschnürung von der alten Narbe aus bedingt, an welcher Einschnürung auch die Kapsel theilnimmt, wie in der Figur gezeichnet. Nachträglich änderte sich nur insofern etwas an diesem Zustande als fer Ciliarfortsatz, welcher dem eingeschnürten Linsenrande entsprach, viel weiter herunter rückte und aus der Reihe der andern hervorragte.

Ein anderer Fall zeigte mir diese Abhebungen nach einem Trauma (Fig. 3, Taf. XIII). Durch ein an das Auge angeflogenes Stuick Holz erlitt Patient eine Verletzung, in Folge deren bei der ersten Vorstellung nur eine Blutung in die vordere Kammer des linken Auges zu sehen war. Als diese sich allmählich aufsaugte, zeigte sich dahinter die I'upille entrundet und nach innen zu ver- 
lagert, so dass tort nur ein sehmaler Theil Iris ubrig geblieben war. Später wurde dieser Theil des Ringes noeh schmäler und hinterliess beim Zurüekziehen an seinem früheren Pupillenrande eine kleine schwarze, nach innen convexe Linie auf der Kapsel als Reste adhärent gebliebenen Irispigmentes. Die Linse selbst war nach aussen davon etwas stärker getrübt, nach innen nur ganz oherflächlich mit feinsten milchweissen Pünktchen besetzt, jedoch nicht so dicht, dass man den Fundus nicht hätte klar durchsehen können. Ich bin nicht vollkommen sicher, ob diese Trîbung der Linse oder der Kapsel angehörte; doch davon später. Ueber diesen Theil hin zogen sich von der Narbe ausgehend 5 ungleich grosse Falten in der beschriebenen Form ron Spiessen mit feinsten Spitzen, die sich in die normal anliegende Kapsel verliefen. Die Sehschärfe war mit $-5 \mathrm{D} \frac{6}{18}$, welche Herabsetzung ieh zum Theil anf die Abhebung zurilckführe. Hier sowie im vorhergehenden Falle erreichten die Falten eine ganz ansehnliche Länge, wie aus der Zeichnung zu sehen.

Merkwiirdig ist in beiden Fällen das Verhalten des Bildes der Abhebung, wenn die Linse durch Accommodationsbewegung ihre Krümmung ändert. Es springen nämlich die Schatten, welche man mit dem lichtscliwachen Spiegel jede Abhebung begleiten sieht, plötzlich mit blitzschneller Bewegung an scheinbar eine andere Stelle, manchmal rasch hinter einander, so. dass das Bild flimmert. Beim Verschioben tes Spiegels dagegen in Ruhelage gehen lie Schatten langsam der Bewegung entsprechend.

Bezüglich der Trübung der sonstigen Linsenoberfläche ist Folgendes, was ihren Sitz anbelangt, ob in der Kapsel, ob epithelial in der Linse, zu überlegen. Es ist zwar schwer, olne Querschnitte der Kapsel und Linse selbst dariber zu entscheiden. Ich möchte aber doch glauben, dass es sich um Trübungen handelt, die in der Kapsel selbst ihren Sitz haben und zwar aus mehrfachen Gründen. Der Erste, welcher über solche Befunde in Präparaten berichtete war H. Miiller, nach welchen die Kapsel Granulationen und körmige Anlagerungen zeigte (welch letzterer Befund sich auch in einigen meiner Präparate bei chronischer Aderhautentzindung etc. fand), welche auch Becker beschrieb, später jedoch für einen Irrthum hielt, da er glaubte, dass 
er diese Befunde hätte ofters antreffen sollen. In letzter Zeit hat Wagenmann bei der Beschreibung einer Kapselkatarakt (v. Graefe's Archiv XXXV. 1, p. 186) jedoch neuerlich beschrieben, dass sich 'in der Kapsel Hohlräume befanden, die mit krümlicher Eiweisssubstanz ausgefüllt waren und bringt hierzu eine Zeichnung.

Ich kann mir ganz gut denken, dass es sich hier um ganz ähnliche Befunde handelt, die makroskopisch als feinste Pünktchen und Inselchen einer milchweissen, fleckigen Trübung imponiren. Gegen den Sitz im Epithel der Linse spricht der Umstand, dass bei solchen Veränderungen des Linsenepithels wohl andere, schwerer Natur hätten nachfolgen sollen, so dass die Linse ihre sonstige Durchsichtigkeit nicht hätte bewahren können.

Anhangsweise möchte ich noch jener Möglichkeit einer Kapselabhebung Frwähnung thun, bei welcher nicht die Kapsel ihrer ganzen Dicke nach sondern nur in einer Lamelle abgehoben erscheint. Die Trennung der Kapsel in ihre Lamellen, die Berger mit hypermangansaurem Kali an der Leiche zeigte, kamn in pathologisch verïnderten Augen gleichfalls, intra vitam, zu Stande kommen.

So steht mir die Tinse eines Auges, das an langdauernder Entziundung im Uvealtract mit ihren Folgen erkrankt war, zur Verfügung, an deren Kapsel sich $\mathrm{Ab}$ spaltungen in mehreren Schichten finden; die Kapsel ist stellenweise wie aufgesplittert, in mehrere ungleich dicke Schichten zertrennt; stellenweise erscheint die oberste Iamelle in grösserer Ausdehnung von den anderen abgehoben, wenn auch die Abhebung nicht eine sehr bedeutende ist. Ich glaube nicht, dass dies eine Leichenerscheinung oder ein Härtungsproduct sei, das man dann wohl bedeutend häufiger antreffen müsste, sondern glaube, dass eine gewisse Sprödigkeit der Kapsel und Tendenz zur Trennung in Lamellen durch stärkere Verbiegungen der ganzen Kapsel im kranken Auge zur Lamellenabhebung Anlass gab (Fig. 5, Taf. XIII). 
$F i g . \pi$
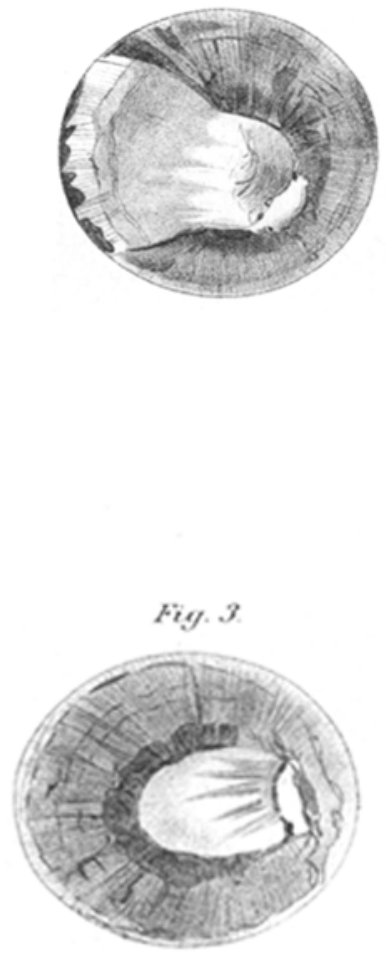

Fiy 5

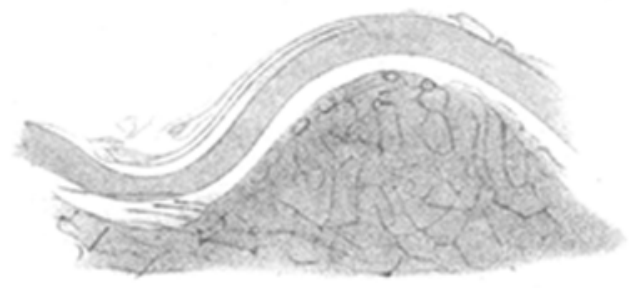

Verlag y. Wilh. Engelmamn in Leiprig. lith.Anst.v.E.A.Funke Leipzig
Fig. 2

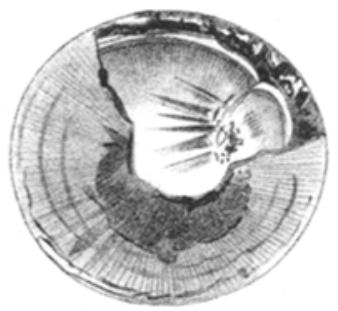

Fig. 4

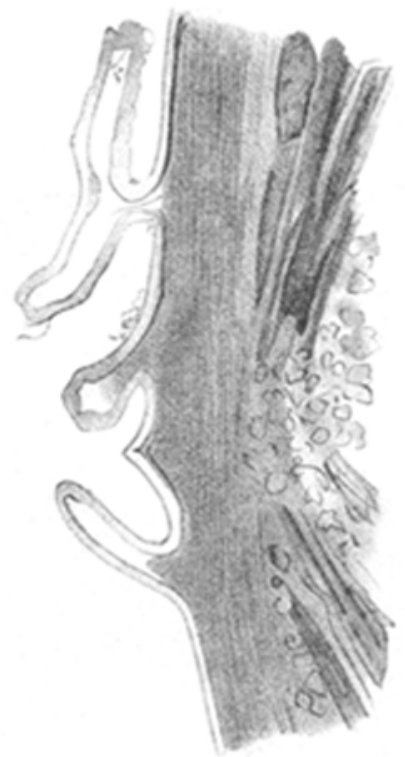

